\title{
Force Constant of the Association Bond in the Formic and Acetic Acid Dimers
}

\author{
J. O. HALFORD \\ Department of Chemistry, University of Michigan, Ann Arbor, Michigan
}

(Received April 4, 1946)

\begin{abstract}
The entropies of the formic and acetic acid dimers are used to find a minimum value for the force constant of the association bond. With the aid of a normal coordinate treatment the probable value is found to be $4 \times 10^{4}$ dynes $/ \mathrm{cm}$. This agrees with a rough estimate from the heat of dimerization. A Raman band in the formic acid spectrum at $200 \mathrm{~cm}^{-1}$ may be due to the association vibrations.
\end{abstract}

$I^{\mathrm{N}}$ $\mathrm{N}$ earlier papers, ${ }^{1}$ the calculation of reasonably accurate en tropies from vapor densities, vapor pressures, and thermal data has been described for the momomers and dimers of formic acid and acetic acid. The dimer entropies, for the hypothetical perfect gases at $25^{\circ} \mathrm{C}$ and atmospheric pressure, were found to be $83.1 \pm 0.1$ and 101.0 \pm 0.3 e.u., respectively, for the two acids.

These entropies may be used to estimate the stretching force constant of the hydrogen bonds by which the two monomer units are thought to be held together in the dimer. The procedure is to find the entropy and frequency of the symmetrical vibration of extension and compression of the association bonds ( $\nu_{1}$ in Fig. 1) and from this frequency to calculate the force constant by means of the familiar relation

$$
2 K=4 \pi^{2} \nu^{2} \mu=2 \pi^{2} \nu^{2} M,
$$

where $M$ is the mass of the monomer.

From the total dimer entropy the contributions of translation and rotation are subtracted to give the internal entropy. It is then assumed that the internal entropy of the monomer is not appreciably changed by combination into the dimer, so that the dimer internal entropy is the sum of twice the monomer internal entropy plus the entropy of six vibrations dependent upon the strength of the association bonds. These vibrations are illustrated in Fig. 1. For all the monomer frequencies except the rotational motion of the hydroxyl group this assumption is acceptable without comment. Extension to include the torsional motion follows at once from the already low entropy ( 0.75 in formic acid) which could only be further lowered to a minor extent by the restrictive forces resulting from the dimerization.

' J. O. Halford, J. Chem. Phys. 9, 859 (1941); 10, 582 (1942).
Table I shows the quantities used to obtain the entropy of the association frequencies. The monomer internal entropy of both acids appears in the first two rows, with the value for hydroxyl torsion set in each case at 0.75 , as originally calculated for formic acid, because of the relatively high probable error in the corresponding quantity (1.2) in the acetic acid calculation. This choice has no effect on the conclusions reached in this paper. The first four rows in the dimer summation in the lower part of the table contain the translational and external rotational entropy, with the constant adjusted to permit direct substitution of the monomer weight in grams for $M$ and of $10^{38}$ times the principal moments of inertia in c.g.s. units for $A, B$, and $C . \sigma$ is the symmetry number.

A model based upon the original structure proposed by Pauling and Brockway, ${ }^{2}$ rather than the newer and less symmetrical one found by

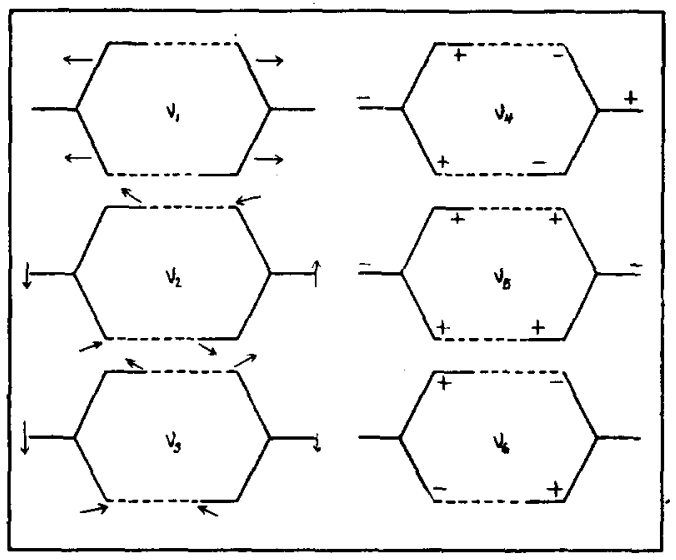

FIG. 1. Vibrations of the formic acid dimer.

${ }^{2}$ L. Pauling and L. O. Brockway, Proc. Nat. Acad. Sci. $20,336(1934)$. 


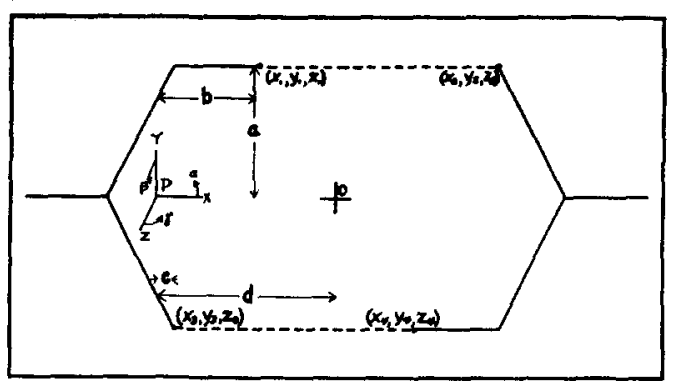

FIG. 2. Coordinates and distances in the formic acid dimer.

Karle and Brockway, ${ }^{3}$ is used to calculate the principal moments of inertia. The rotational entropy does not vary appreciably with minor changes in the model. Dimensions used are $(\mathrm{H}-\mathrm{C})=1.09, \quad(\mathrm{C}-\mathrm{O})=(\mathrm{C}=\mathrm{O})=1.28, \quad$ and $(\mathrm{C}-\mathrm{C})=1.54 \mathrm{~A}$ units, with the $\mathrm{O}-\mathrm{C}-\mathrm{O}$ angle set at the rounded value of $120^{\circ}$. The oxygen atoms of the individual monomer units are taken to be linked through the bonding hydrogen at a distance of $2.67 \mathrm{~A}$ units, with the hydrogen atom placed at 1.00 unit from the nearer oxygen. For the principal axes it is accurate enough to take the common bisector of the two $\mathrm{O}-\mathrm{C}-\mathrm{O}$ angles and its perpendiculars in and at right angles to the molecular plane. The two axes in the molecular plane are thus placed slightly out of their true positions because of the location of the hydroxyl hydrogens.

By analogy to the properties of ordinary valence bonds, $\nu_{1}$ and $\nu_{3}$ should represent considerably higher frequencies than the remaining four vibrations. It is safe to assume therefore that the entropy of the first frequency is less than one-sixth of the total shown in Table I for the association vibrations. Thus $S_{1}$ is less than 2.53 e.u. for formic acid, and, from the Einstein function, $\nu_{1}$ is greater than $162 \mathrm{~cm}^{-1}$. The corresponding value for $K$ is $1.7 \times 10^{4}$ dynes $/ \mathrm{cm}$. Similarly, for acetic acid, $S_{1}$ is less than $3.09, \nu_{1}$ is greater than 121 , and $K$ is above $1.1 \times 10^{4}$. The minimum is therefore determined by the more accurate formic acid entropy.

The probable value is considerably larger because at least one of the bending motions would be expected to have a much larger entropy, leaving less for $\nu_{1}$. It is more difficult to estimate a maximum. From symmetry considerations $\nu_{1}$

${ }^{3}$ J. Karle and L. O. Brockway, J. Am. Chem. Soc. 66, 574 (1944). should be active in Raman scattering but there is no way of predicting how intense the effect should be. It seems improbable, even if the intensity is low, that the Raman line would have escaped detection if the frequency were greater than $250 \mathrm{~cm}^{-1}$, for which $K$ for formic acid is $4.2 \times 10^{4}$. For acetic acid the constant derived from this frequency would be higher.

The result of this estimate, $K=3.0 \pm 1.3 \times 10^{4}$, can be supported in two independent ways, and, in addition, by a more detailed interpretation of the entropy by means of the normal coordinate method. A Raman band has been reported for liquid formic acid at $180 \pm 135$ by Edsall, ${ }^{4}$ at 200 by Kohlrausch, Koppl, and Pongratz, ${ }^{5}$ and at $197 \mathrm{~cm}^{-1}$ by Morino and Mizushima. ${ }^{6}$ According to Hibben ${ }^{7}$ this may be analogous to a similar scattering observed for water, for which he suggests as a possible cause a hindered rotation in the liquid state. It is also possible that the effect may be due to the vibrations under discussion here. If so, a frequency of $200 \mathrm{~cm}^{-1}$ would indicate for the hydrogen bond a stretching force constant of $2.7 \times 10^{4}$ dynes $/ \mathrm{cm}$.

A second independent supporting estimate is based upon the assumption that the ratio of force constant to energy of dissociation is about the same for the association bond as for ordinary hydrogen valence bonds. For the $\mathrm{CH}, \mathrm{OH}, \mathrm{ClH}$,

TABLE I. Entropy of association vibrations at $25^{\circ} \mathrm{C}$.

\begin{tabular}{lcr}
\hline & Formic acid & Acetic acid \\
\hline Vibration & Monomer & \\
Hydroxyl torsion & 0.60 & 3.60 \\
Methyl torsion & -75 & .75 \\
Internal entropy & 1.35 & 2.99 \\
& & 7.34 \\
Constant & Dimer & \\
$\frac{3}{2} R \ln 2 M$ & 49.87 & 49.87 \\
$\frac{1}{2} R \ln A B C$ & 13.48 & 14.27 \\
$-R \ln \sigma$ & 3.22 & 5.04 \\
Internal, monomer & -1.38 & -1.38 \\
Association bonds & 2.70 & 14.68 \\
& 15.21 & 18.56 \\
& 83.10 & 101.04 \\
\hline
\end{tabular}

${ }^{4}$ J. T. Edsall, J. Chem. Phys. 4, 1 (1937).

${ }^{5}$ K. W. F. Kohlraúsch, F. Koppl, and A. Pongratz, Zeits. f. physik. Chemie 21B, 242 (1933).

${ }_{6}$ Y. Morino and S. Mizushima, Sci. Pap. Inst. Phys. Chem. Research ('Tokyo) 32, 33 (1937).

${ }^{7} \mathrm{~J}$. H. Hibben, The Raman Effect and Its Chemical Applications (Reinhold Publishing Corporation, New York, $1939)$, p. 180 . 
TABLE II. Molecular constants of monomer units.

\begin{tabular}{ccc}
\hline & Formic acid & Acetic acid \\
\hline $10^{22} M$ & 0.7593 & 0.9906 \\
$10^{38} D$ & 0.7906 & 1.6053 \\
$10^{38} E$ & 0.6685 & 0.7214 \\
$10^{38} F$ & 0.1221 & 0.9365 \\
$10^{8} a$ & 1.109 & 1.109 \\
$10^{8} b$ & 1.183 & 1.675 \\
$10^{8} c$ & 0.183 & 0.675 \\
$10^{8} d$ & 1.518 & 2.010 \\
\hline
\end{tabular}

and $\mathrm{BrH}$ bonds this ratio has an average value near 5.7 dynes $/ \mathrm{cm} / \mathrm{cal}$. The heat of dissociation of the formic acid dimer, 7150 cal. per hydrogen bond, is taken to be near enough to the energy of dissociation and is multiplied by the above ratio to give $K=4.1 \times 10^{4}$.

Finally, to provide support for the assumption that the entropy of $\nu_{1}$ should be considerably less than the average for the six association frequencies, a normal coordinate treatment is undertaken to determine what spread of frequencies is consistent with a simplified but reasonable potential function. In addition to its application to the present problem, this study of a planar system with a center of symmetry but no other symmetry elements is of interest for itself.

The molecular model is shown in Fig. 2 which gives part of the symbolism including the coordinates applied to one monomer unit in setting up the initial equations. Because it has been assumed that the association vibrations are independent of other molecular motions, the model consists of two rigid bodies vibrating against each other. For such a system the kinetic energy may be conveniently expressed in terms of the translation and rotation of the individual rigid bodies. $X, Y$, and $Z$ represent linear displacements of the centers of mass, while $\alpha, \beta$, and $\gamma$ are angular displacements about the principal axes.

The kinetic energy is given by the following equation :

$$
\begin{aligned}
2 T & =M\left(\dot{X}_{1}{ }^{2}+\dot{Y}_{1}{ }^{2}+\dot{Z}_{1}{ }^{2}+\dot{X}_{2}{ }^{2}+\dot{Y}_{2}{ }^{2}+\dot{Z}_{2}{ }^{2}\right) \\
& +D\left(\dot{\alpha}_{1}^{2}+\dot{\alpha}_{2}^{2}\right)+E\left(\dot{\beta}_{1}^{2}+\dot{\beta}_{2}{ }^{2}\right)+F\left(\dot{\gamma}_{1}{ }^{2}+\dot{\gamma}_{2}{ }^{2}\right) .
\end{aligned}
$$

Subscripts distinguish the two monomer units. $D, E$, and $F$ are moments of inertia about the axes $P Z, P X$, and $P Y$, respectively, of Fig. 2 .

For the potential energy,

$$
\begin{array}{r}
2 V=K\left[\left(x_{1}-x_{2}\right)^{2}+\left(x_{3}-x_{4}\right)^{2}\right] \\
+2 k\left(y_{1}{ }^{2}+y_{2}{ }^{2}+y_{3}{ }^{2}+y_{4}{ }^{2}\right. \\
\left.+z_{1}{ }^{2}+z_{2}{ }^{2}+z_{3}{ }^{2}+z_{4}{ }^{2}\right) .
\end{array}
$$

TABLE III. Equations for association frequencies.

\begin{tabular}{cc}
\hline \hline $10^{-22 \lambda}$ (formic acid) & $10^{-22 \lambda}$ (acetic acid) \\
\hline $5.268 K$ & $4.038 K$ \\
$2.497 k$ & $2.900 k$ \\
$6.223 K+3.625 k$ & $3.065 K+4.063 k$ \\
$21.941 k$ & $5.645 k$ \\
$26.605 k$ & $9.593 k$ \\
$4.228 k$ & $4.193 k$ \\
\hline
\end{tabular}

Here the subscripts indicate the terminal points of the association bonds, with even numbers applying to one monomer unit and odd numbers to the other, as shown in Fig. 2. To obtain Eq. (3) the general quadratic potential function has been simplified by eliminating all cross products except the $x_{1} x_{2}$ and $x_{3} x_{4}$ terms and reducing the number of constants to two. For this form, $K$ is evidently the stretching valence force constant, while $k$, which is concerned with lateral displacements, must be a bending force constant. Bending constants defined in this way are about onefifth to one-tenth as large as stretching constants, and may be converted to angular valence force constants by multiplying by the squares of the appropriate bond lengths. When, as in the present problem, the same constant is used for all the angular displacements, the factor required for this conversion is not evident, and the relation to any specific angular valence force constant is not definite. The product $k\left(b^{2}+c^{2}\right)$ in Eqs. (7) and (8) should have about the right magnitude for an angular valence force constant because $b$ and $c$ are of the correct order for interatomic distances. Equations (8) show the correct form for the angular vibration between like rotators with the reduced moment of inertia $D / 2$, etc. These solutions were put into this form by introducing the factor $2 k$ rather than $k$ into Eq. (3).

Six of the twelve coordinates in the kinetic energy equation are eliminated by means of the following linear and angular momentum conditions :

$$
\begin{array}{cc}
\dot{X}=\dot{X}_{1}=-\dot{X}_{2} & \dot{\beta}=\dot{\beta}_{1}=-\dot{\beta}_{2} \\
\dot{Y}=\dot{Y}_{1}=-\dot{Y}_{2} & 2 M d \dot{Y}=D\left(\dot{\alpha}_{1}+\dot{\alpha}_{2}\right) \\
\dot{Z}=\dot{Z}_{1}=-\dot{Z}_{2} & 2 M d \dot{Z}=F\left(\dot{\gamma}_{1}+\dot{\gamma}_{2}\right) .
\end{array}
$$

The substitutions $\alpha_{1}=\theta_{1}+\theta_{2}, \alpha_{2}=\theta_{1}-\theta_{2}, \gamma_{1}=\varphi_{1}$ $+\varphi_{2}$ and $\gamma_{2}=\varphi_{1}-\varphi_{2}$ are then made, leading to the expression

$$
\begin{aligned}
2 T=2 M \dot{X}^{2}+2 D\left(1+D / M d^{2}\right) \dot{\theta}_{1}{ }^{2}+2 D \dot{\theta}_{2}{ }^{2} \\
+2 E \dot{\beta}^{2}+2 F\left(1+F / M d^{2}\right) \dot{\varphi}_{1}{ }^{2}+2 F \dot{\varphi}_{2}{ }^{2} .
\end{aligned}
$$


TaBLE IV. Trial solutions.

\begin{tabular}{|c|c|c|}
\hline \multicolumn{3}{|c|}{ Formic acid, $K=5 k$} \\
\hline$\nu / \nu_{1}$ & $\nu\left(\mathrm{cm}^{-1}\right)$ & $S_{298}$ \\
\hline $\begin{array}{l}1.000 \\
0.308 \\
1.148 \\
0.913 \\
1.005 \\
0.401\end{array}$ & $\begin{array}{r}229 \\
71 \\
263 \\
209 \\
229 \\
92\end{array}$ & $\begin{array}{l}1.89 \\
4.13 \\
1.65 \\
2.06 \\
1.89 \\
3.62\end{array}$ \\
\hline \multicolumn{2}{|c|}{$K=3.5 \times 10^{4}$} & 15.24 \\
\hline \multicolumn{3}{|c|}{ Formic acid, $K=10 k$} \\
\hline $\begin{array}{l}1.000 \\
0.218 \\
1.118 \\
0.645 \\
0.711 \\
0.283\end{array}$ & $\begin{array}{r}293 \\
64 \\
328 \\
189 \\
208 \\
83\end{array}$ & $\begin{array}{l}1.46 \\
4.35 \\
1.28 \\
2.24 \\
2.06 \\
3.82\end{array}$ \\
\hline \multicolumn{2}{|c|}{$K=5.7 \times 10^{4}$} & 15.21 \\
\hline \multicolumn{3}{|c|}{ Acetic acid, $K=5 k$} \\
\hline $\begin{array}{l}1.000 \\
0.379 \\
0.980 \\
0.529 \\
0.689 \\
0.456\end{array}$ & $\begin{array}{r}193 \\
73 \\
189 \\
102 \\
133 \\
88\end{array}$ & $\begin{array}{l}2.21 \\
4.07 \\
2.24 \\
3.42 \\
2.91 \\
3.70\end{array}$ \\
\hline \multicolumn{2}{|c|}{$K=3.3 \times 10^{4}$} & 18.55 \\
\hline \multicolumn{3}{|c|}{ Acetic acid, $K=10 k$} \\
\hline $\begin{array}{l}1.000 \\
0.268 \\
0.927 \\
0.374 \\
0.482 \\
0.322\end{array}$ & $\begin{array}{r}246 \\
66 \\
228 \\
92 \\
120 \\
79\end{array}$ & $\begin{array}{l}1.76 \\
4.27 \\
1.89 \\
3.61 \\
3.11 \\
3.92\end{array}$ \\
\hline \multicolumn{2}{|c|}{$K=5.3 \times 10^{4}$} & 18.56 \\
\hline
\end{tabular}

For the transformation of the potential energy the required equations are

$$
\begin{array}{lll}
x_{1}=X-a \alpha_{1} & y_{1}=b \alpha_{1} & z_{1}=a \beta+b \gamma_{1} \\
x_{2}=-X-a \alpha_{2} & y_{2}=-c \alpha_{2} & z_{2}=-a \beta-c \gamma_{2} \\
x_{3}=X+a \alpha_{1} & y_{3}=c \alpha_{1} & z_{3}=-a \beta+c \gamma_{1} \\
x_{4}=-X+a \alpha_{2} & y_{4}=-c \alpha_{2} & z_{4}=a \beta-b \gamma_{2}
\end{array}
$$

together with the definition equations for $\theta_{1}, \theta_{2}$, $\varphi_{1}$, and $\varphi_{2}$. After the transformation the potential energy has the form

$$
\begin{aligned}
2 V=8 K X^{2}+ & 4 k\left(b^{2}+c^{2}\right)\left(\theta_{1}{ }^{2}+\varphi_{1}{ }^{2}+\varphi_{2}{ }^{2}\right) \\
& +4\left[2 K a^{2}+k\left(b^{2}+c^{2}\right)\right] \theta_{2}{ }^{2} \\
& +8 k a(b-c) \beta \varphi_{2}+8 k a^{2} \beta^{2} .
\end{aligned}
$$

The solutions for $\lambda=4 \pi^{2} \nu^{2}$ for the first four vibrations of Fig. 1 take the forms

$$
\begin{gathered}
\lambda_{1}=4 K / M \quad \lambda_{2}=2 k\left(b^{2}+c^{2}\right) /\left[D\left(1+D / M d^{2}\right)\right] \\
\lambda_{3}=\left[4 K a^{2}+2 k\left(b^{2}+c^{2}\right)\right] / D \\
\lambda_{4}=2 k\left(b^{2}+c^{2}\right) /\left[F\left(1+F / M d^{2}\right)\right] .
\end{gathered}
$$

For $\lambda_{5}$ and $\lambda_{6}$,

$$
\left|\begin{array}{cc}
E \lambda-4 k a^{2} & -2 k a(b-c) \\
-2 k a(b-c) & F \lambda-2 k\left(b^{2}+c^{2}\right)
\end{array}\right|=0 .
$$

The masses, moments of inertia, and distances required for numerical solution of the above equations are collected in Table II. The moments $D, E$, and $F$ have been calculated with the aid of the dimensions listed earlier for the dimer and are subject to a similar but relatively larger error because of the deviation of the selected rotational axes from the principal axes. The error is again taken to be negligible. The distances $a, b$, and $c$ are coordinates referred to the axes $P X$ and $P Y$, as shown in Fig. 2, and $d$ is the distance between $O$, the center of mass of the dimer, and $P$, that of the monomer unit.

Substitution of the constants of Table II into Eqs. (8) and (9) yields the simplified expressions of Table III.

In principle the two potential constants could be found by trial from the two entropy totals of Table I, but the result so obtained would have little meaning because of the relatively large uncertainty in the acetic acid entropy. It seems better to assign a reasonable value to $K / k$, to evaluate from this the ratios $\lambda / \lambda_{1}$ and $\nu / \nu_{1}$, and to find by trial from the entropy-frequency curve a set of frequencies in the corresponding ratios which will yield the correct entropy. Table IV shows in successive columns the frequency"ratios, the frequencies, and the entropy summation for four such trial solutions.

The assumed ratios of 5 and 10 for $K / k$ are of the order usually found for ordinary valence bonds. For each ratio the spread of the calculated frequencies is several-fold and $\nu_{1}$ appears at or near the maximum, accounting for considerably less than one-sixth of the entropy of the association frequencies. It is therefore probable that $\nu_{1}$ for formic acid lies in the region between 200 and $250 \mathrm{~cm}^{-1}$ and that $K$ is not far from $4 \times 10^{4}$ dynes/cm.

In the absence of a more accurate method of evaluation this provides a satisfactory approximation to the stretching potential constant of the association bond in the carboxylic acid dimer. 